\title{
Radiosurgery reirradiation for high-grade glioma recurrence: a retrospective analysis
}

\author{
Valentina Pinzi ${ }^{1} \cdot$ Chiara Orsi $^{2} \cdot$ Marcello Marchetti $^{1} \cdot$ Ida Maddalena Milanesi $^{1}$. \\ Livia Corinna Bianchi ${ }^{3}$ - Francesco DiMeco ${ }^{4}$. Valeria Cuccarini ${ }^{5}$. \\ Mariangela Farinotti $^{6}$ - Paolo Ferroli ${ }^{4}$ - Gaetano Finocchiaro ${ }^{7}$ Angelo Franzini ${ }^{4}$. \\ MariaLuisa Fumagalli ${ }^{8} \cdot$ Antonio Silvani $^{7} \cdot$ Laura Fariselli $^{1}$
}

Received: 20 January 2015/Accepted: 13 March 2015/Published online: 25 March 2015

(C) Springer-Verlag Italia 2015

\begin{abstract}
Despite various treatment strategies being available, recurrent high-grade gliomas (r-HGG) are difficult to manage. To obtain local control, radiosurgery (SRS) reirradiation has been considered as potential treatment. In the present study, a retrospective analysis was performed on r-HGG patients treated with salvage single- (s-SRS) or multi-fraction SRS (m-SRS). The aim of this study was to evaluate the effectiveness of salvage SRS in terms of overall survival (OS); toxicity was analyzed as well. Between 2004 May and 2011 December, 128 r-HGG patients (161 lesions) treated with CyberKnife ${ }^{\circledR}$ SRS reirradiation were retrospectively analyzed. Toxicity was graded
\end{abstract}

Valentina Pinzi

valentinapinzi@yahoo.it; pinzi@istituto-besta.it

1 Radiotherapy Unit, Neurosurgery Department, Fondazione IRCCS Istituto Neurologico Carlo Besta, Via Celoria 11, 20133 Milan, MI, Italy

2 Section of Biostatistics and Clinical Epidemiology, Department of Public Health, Experimental and Forensic Medicine, University of Pavia, Via Severino Boezio 24, 27100 Pavia, Italy

3 CyberKnife Center, CDI (Centro Diagnostico Italiano), Via Saint Bon 20, 20147 Milan, Italy

4 Neurosurgery Department, Fondazione IRCCS Istituto Neurologico Carlo Besta, Via Celoria 11, 20133 Milan, Italy

5 Neuroradiology Unit, Neurosurgery Department, Fondazione IRCCS Istituto Neurologico Carlo Besta, Via Celoria 11, 20133 Milan, Italy

6 Unit of Neuroepidemiology, Fondazione IRCCS Istituto Neurologico Carlo Besta, Via Celoria 11, 20133 Milan, Italy

7 Unit of Neuro-oncology, Fondazione IRCCS Istituto Neurologico Carlo Besta, Via Celoria 11, 20133 Milan, Italy

8 Health Department, Fondazione IRCCS Istituto Neurologico Carlo Besta, Via Celoria 11, 20133 Milan, Italy according to Radiation Therapy Oncology Group and by Common Terminology Criteria for Adverse Events v.3 criteria. OS from the diagnosis date and OS from reirradiation were estimated using the Kaplan-Meier method. Median follow-up was 9 months (range 15 days82 months). All patients completed SRS without highgrade toxicity. Radiation necrosis was observed in seven patients $(6 \%)$ with large volume lesions. The median survival from initial diagnosis was 32 months. The 1-, 2-, and 3-years survival rates from diagnosis were 95, 62, and $45 \%$ respectively. Median survival following SRS was 11.5 months. The 1-, 2-, and 3-years survival rate following SRS was 48, 20, and $17 \%$ respectively. On multivariate analysis, age $<40$ years, salvage surgery before SRS, and other post-SRS therapies (second-line chemotherapy and/or surgery) were found to significantly improve survival $(p=0.03)$. SRS represents a safe and feasible option to treat r-HGG patients with low complication rates and potential survival benefit.

Keywords Single-fraction radiosurgery $\cdot$ Multi-fraction radiosurgery $\cdot$ Reirradiation $\cdot$ Recurrent high-grade glioma

\section{Introduction}

A number of treatment options are available for recurrent high-grade gliomas (r-HGG), including surgery, secondline chemotherapy, targeted agents therapy, and reirradiation. All strategies involve the high probability of controversial therapy-related side effects and may be feasible only within a subgroup of patients. Since approximately $80 \%$ of cases of high-grade gliomas (HGG) recur within $2 \mathrm{~cm}$ of the primary tumors, where the highest doses of radiotherapy (RT) have already been delivered in the 
primary adjuvant or definitive setting [1], reirradiation is often restricted, because of the high risk of radiation toxicity.

To overcome this limitation minimally invasive treatments aiming at local control of disease, such as brachytherapy [2, 3], intermediate-frequency alternating electric fields [4], single-fraction radiosurgery (s-SRS) [5, 6], and multi-fraction radiosurgery (m-SRS) [6, 7] have been proposed. Radiosurgery (SRS) involves a brief, intensified regimen of tightly focused external photon beams targeting one or more discrete intracranial lesions. This peculiarity, together with the improvements in set-up accuracy, may permit a reduction in the prescribed dose volume, and may therefore be advantageous over standard RT. In the attempt to spare adjacent normal brain tissue and organs at risk of therapy-related side effects either s-SRS or $\mathrm{m}$-SRS may be a safe and effective option delivering high radiation doses to small target volumes of previously irradiated tissue [8]. The ability of brain tissue to recover from sub-lethal damage - the principal factor determining the size of the reirradiation dose-was found to be dependent upon the initial biologically effective dose (BED), as well as the time interval between the initial exposure and reirradiation $[9,10]$. Although brain, which is considered a late responding tissue, is more likely to be damaged by higher doses per fraction, SRS is more effective in sparing the surrounding tissue, thus counterbalancing the impact of high doses and reducing the grade and the rate of radiationinduced toxicity [11]. Fractionated SRS is more effective in sparing adjacent normal tissue since it exploits the radiobiological mechanism of recovery from sub-lethal damage. Theoretically, this latter modality may permit the safe treatment of larger target areas.

The current study evaluates the clinical results of SRS (both single- and multi-fraction) in 128 patients who had already undergone primary RT as adjuvant or definitive treatment in response to the question of whether different patterns of recurrences and features of treatment may affect survival. The object of the present retrospective study is to evaluate the effectiveness of salvage SRS in terms of overall survival; including an analysis of toxicity.

\section{Patients and methods}

Following the approval by Ethic Committee Board a retrospective review was performed. All analyzed patients had signed a written informed consent for the scientific use of medical data.

Between 2004 May and 2011 December, 128 consecutive patients with r-HGG lesions were treated with salvage radiosurgery at our Institute in collaboration with CDI (Centro diagnostico italiano, Milan), using CyberKnife ${ }^{\circledR}$.
The initial histological diagnosis was glioblastoma (GBM) for 88 patients (69 \%), and WHO grade III gliomas for 40 patients $(31 \%)$. Based on CT or MRI postoperative imaging, resection type was defined as follows: gross total resection, performed in $30 \%$ of evaluable patients; subtotal resection in $60 \%$; biopsy in $10 \%$, while the extent of surgery was unspecified in 11 patients $(8 \%$ of global patient population). The $8 \%$ of patients with unspecified extent of resection were excluded from the statistical analysis related to type of resection. All patients received a standard course of RT (mean dose $58 \mathrm{~Gy}$, range 40-60 Gy). After 2004, all GBM patients received concomitant temozolomide as well. Recurrence was defined as the presentation of new or increasingly contrast-enhanced lesions in T1-weighted magnetic resonance imaging (MRI) after standard fractionated RT; recurrence was considered infield when it was within $2 \mathrm{~cm}$ of the primary target volume and distant when it was beyond. The median time of progression after initial radiotherapy treatment was 11 months with a range between 2 and 171 months.

\section{Radiosurgery planning and delivery}

Patients underwent localizing contrast-enhanced computed tomography (CT) and post-contrast volumetric three-dimensional T1-weighted MRI, in either 1 or $1.5 \mathrm{~mm}$ thicknesses, and then were transferred to the planning system. Following image fusion, the target volume was defined by the contrast-enhanced marginless tumor edges. An inverse planning method using non-isocentric technique was used in all cases (Multiplan Accuray Inc., Sunnyvale, CA, USA) (Fig. 1). The median planning tumor volume (PTV) of treatment was $5.2 \mathrm{~cm}^{3}$ (range $0.14-120 \mathrm{~cm}^{3}$ ); the median PTV was $2 \mathrm{~cm}^{3}$ (range $0.14-83 \mathrm{~cm}^{3}$ ) for s-SRS and $10 \mathrm{~cm}^{3}$ (range $0.63-120 \mathrm{~cm}^{3}$ ) for $\mathrm{m}$-SRS. Depending on target volume and proximity to eloquent areas, the treatment was delivered in single- or multi-fractions. The prescribed dose for reirradiation was chosen with respect to prior radiation volume, total dose, and interval between treatments. The prescribed reirradiation dose was evaluated in relation to the final radiation isodose distribution. The median prescription dose was 15 Gy (range 6-22 Gy) for s-SRS; 23 Gy (range 12-28 Gy), with a median number of 3 fractions, for m-SRS. The median prescription isodose was $80 \%$ (range 65-89\%). Ninety-nine patients received SRS once, 25 twice, and 4 patients three times. SRS treatment parameters are summarized in Table 1.

\section{Toxicity}

Central nervous system toxicity was defined as any new or aggravated neurological signs or symptoms probably related to radiosurgery treatment. 


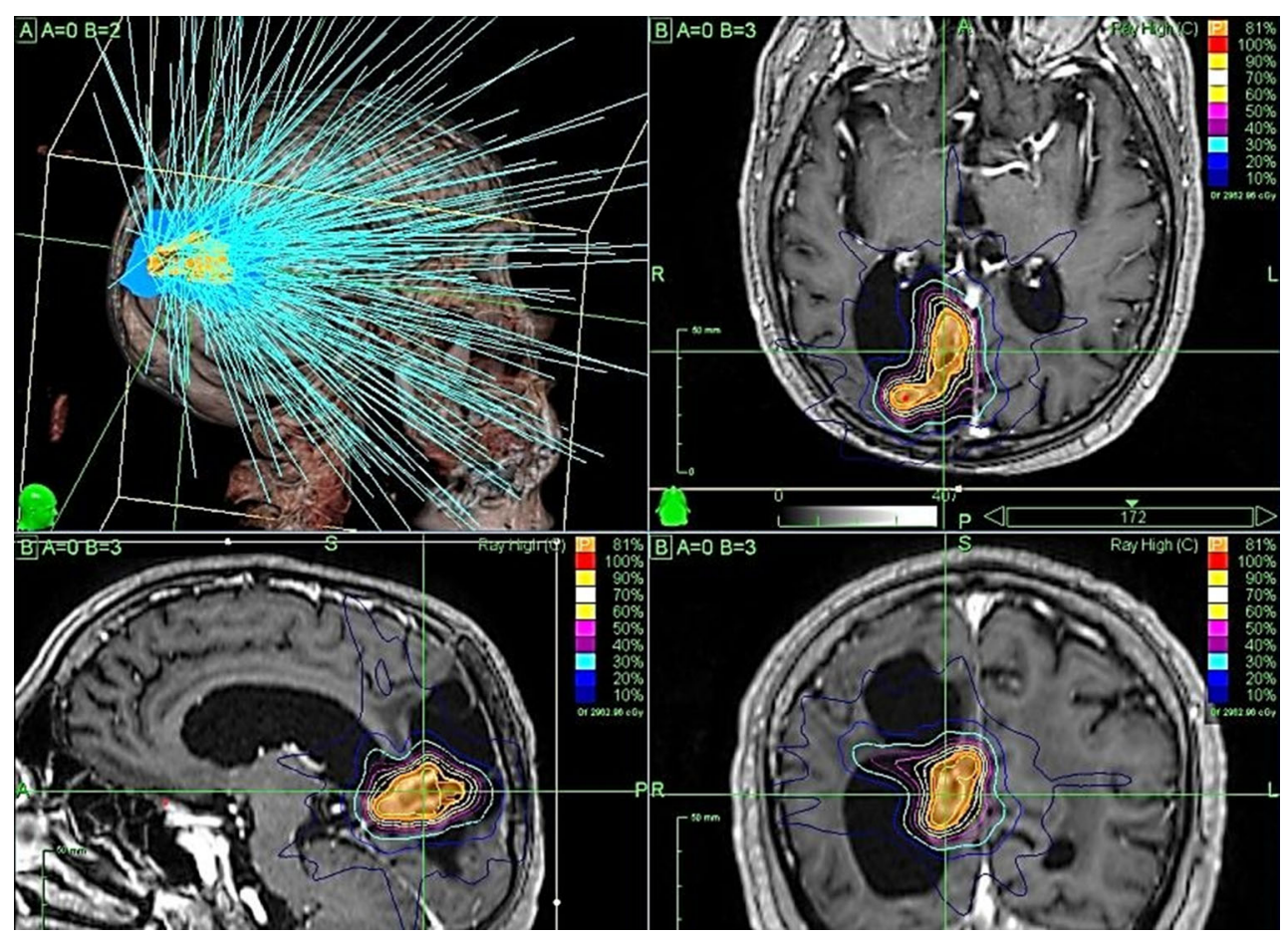

Fig. 1 A CyberKnife three-dimensional rendering of a non-coplanar beam arrangement on T1-weighted magnetic resonance imaging (MRI)

Table 1 Treatment parameters

\begin{tabular}{ll}
\hline SRS (radiosurgery) type & $n(\%)$ \\
s-SRS (single-fraction SRS) & $42(33)$ \\
m-SRS (multi-fraction SRS) & $86(67)$ \\
Recurrence pattern lesions & $n(\%)$ \\
Total & $161(100)$ \\
Infield & $117(73)$ \\
Distant & $44(27)$ \\
Dose of s-SRS (single-fraction SRS) & $\mathrm{Gy}$ \\
Median & 15 \\
Range & $6-22$ \\
Dose of m-SRS (multi-fraction SRS) & $\mathrm{Gy}$ \\
Median & 23 \\
Range & $12-28$ \\
Planning target volume of s-SRS & $\mathrm{cm}^{3}$ \\
Median & 2 \\
Range & $0.14-83$ \\
Planning target volume of m-SRS & $\mathrm{cm}^{3}$ \\
Median & 11 \\
Range & $0.63-121$ \\
Isodose prescription & $\%$ \\
Median & 80 \\
Range & $65-89$ \\
\hline
\end{tabular}

Toxicity was graded according to Radiation Therapy Oncology Group (RTOG) [12] and by Common Terminology Criteria for Adverse Events (CTCAE) version 3 criteria [13].

\section{Other therapies}

Prior to radiosurgery, most patients had undergone other salvage therapies: second-line chemotherapy was administered in 60 patients (47\%), while 20 patients (16\%) had undergone surgical resection.

\section{Statistical analysis}

Descriptive statistics were calculated by means of absolute and relative frequencies for categorical variables and mean standard deviation, median, and range for continuous variables. The analyzed variables are selected based on literature data. The selected variables are detailed in Table 2.

Overall survival (OS) from the diagnosis date (defined as the time between initial surgery and death) and OS from reirradiation (defined as the time between end of reirradiation and death) were estimated using the Kaplan-Meier 
Table 2 Univariate and multivariate survival from diagnosis and reirradiation

\begin{tabular}{|c|c|c|c|c|c|c|c|c|}
\hline \multirow[t]{3}{*}{ Variables } & \multirow{3}{*}{$\begin{array}{l}\text { From } \\
\text { Univariate } \\
p\end{array}$} & \multicolumn{3}{|c|}{ Diagnosis } & \multirow{3}{*}{$\begin{array}{l}\text { From } \\
\text { Univariate } \\
p\end{array}$} & \multirow{2}{*}{\multicolumn{3}{|c|}{$\frac{\text { Reirradiation }}{\text { Multivariate }}$}} \\
\hline & & \multicolumn{3}{|c|}{ Multivariate } & & & & \\
\hline & & HR & $95 \% \mathrm{CI}$ & $p$ & & HR & $95 \% \mathrm{CI}$ & $p$ \\
\hline Age at diagnosis ( $<40$ vs $\geq 40$ years) & 0.0004 & 1.90 & $1.18-3.06$ & 0.008 & 0.1738 & - & - & N.A. \\
\hline Age at reirradiation ( $<40$ vs $\geq 40$ years) & 0.0144 & - & - & N.A. & 0.0460 & 1.35 & $0.86-2.11$ & 0.189 \\
\hline Histology (GBM vs grade III) & 0.0000 & 0.89 & $0.19-4.11$ & 0.883 & 0.0103 & 0.66 & $0.15-2.86$ & 0.583 \\
\hline $\mathrm{RT}$ dose $(<60$ vs $=60 \mathrm{~Gy})$ & 0.0001 & 1.34 & $0.75-2.41$ & 0.314 & 0.0084 & 0.80 & $0.41-1.53$ & 0.510 \\
\hline RPA classes (1-2 vs 4-6-7) & 0.0000 & 1.50 & $0.32-7.03$ & 0.605 & 0.0099 & 1.32 & $0.31-5.63$ & 0.705 \\
\hline Other therapies ${ }^{\mathrm{a}}$ before SRS (no vs yes) & 0.0173 & 0.74 & $0.49-1.13$ & 0.176 & 0.6135 & & & - \\
\hline Salvage surgery before SRS (no vs yes) & 0.0278 & 0.51 & $0.30-0.89$ & 0.019 & 0.3813 & & & - \\
\hline Other therapies ${ }^{\mathrm{a}}$ after SRS (no vs yes) & 0.6165 & & & - & 0.0307 & 0.66 & $0.44-0.98$ & 0.043 \\
\hline Frontal site at diagnosis (no vs yes) & 0.0503 & & & - & 0.4175 & & & - \\
\hline Frontal site at SRS (no vs yes) & 0.0812 & & & - & 0.4479 & & & - \\
\hline Number of SRS fractions ( 1 vs $>1)$ & 0.2750 & & & - & 0.8514 & & & - \\
\hline Number of SRS lesions ( 1 vs $>1$ ) & 0.3899 & & & - & 0.7964 & & & - \\
\hline Lesion volume ( $<7$ vs $\left.\geq 7 \mathrm{~cm}^{3}\right)$ & 0.9840 & & & - & 0.1335 & & & - \\
\hline
\end{tabular}

Bold values are statistically significant

$p p$ value, $H R$ hazard ratio, $C I$ confidence interval, N.A. not available, N.S. not significant, $G B M$ glioblastoma multiforme, $R T$ conformal external beam radiotherapy, $R P A$ recursive partitioning analysis, $S R S$ radiosurgery

a Other therapies before/after SRS: chemotherapy and/or surgery performed before or after SRS reirradiation

method. Survival for different subgroups was also estimated using the Kaplan-Meier method and compared by means of the log-rank test. All statistically significant variables $(p<0.05)$ obtained by the univariate analysis were included in a Cox proportional hazard rate model.

\section{Results}

\section{Patient characteristics}

Between 2004 and 2011, a total of 128 patients (161 lesions) received SRS treatment with CyberKnife ${ }^{\circledR}$ and their follow-up was performed at our Institute.

The median time of progression after initial radiotherapy treatment was 11 months with a range of 2 and 171 months. One hundred and seventeen $(73 \%)$ recurrent lesions were infield and $44(27 \%)$ distant. SRS was performed after a median period of 15 months from standard RT (range 6-171 months).

At the time of SRS, the Karnofsky performance score (KPS) was $\geq 70$ for all patients; mean age was 51 years (range 18-79 years). Based on r-HGG classes [14], 18 patients were in RPA 1; 20 in RPA 2; 31 in RPA 4; 41 in RPA 6, and 18 in RPA 7. Disease progression following salvage SRS was treated with surgery in 17 of evaluable patients $(13 \%)$ and chemotherapy in $31(25 \%)$ patients, under different regimens. In $29(28 \%)$ patients with distant progression, radiosurgery was delivered more than one time. The characteristics of patients and therapies are summarized in Table 3.

\section{Survival}

By the end of study, 91 patients had died of tumor progression and 37 patients were still alive. Median follow-up was 9 months (range 15 days-82 months).

\section{Survival from diagnosis (first surgery)}

The median survival for the whole group was 32 months (95\% CI 25-43, SD 3.6) (Fig. 2). Survival rates from diagnosis for 1,2 , and 3 years were 95,62 , and $45 \%$, respectively. For patients younger than 40 years at the time of diagnosis the median survival was 65 months $(95 \% \mathrm{CI}$ $35-99$, SD \pm 1.8 ); for patients $\geq 40$ years was 25 months (95\% CI 22-32, SD \pm 1.6 ). Median survival for GBM and grade III glioma group was 25 months (95\% CI 22-31, $\mathrm{SD} \pm 1.7)$ and 65 months (95\% CI 43-111, SD \pm 1.8 ), respectively.

Under univariate analysis, there was a statistically significant improvement in survival for patients $<40$ years old $(p=0.0004)$, grade III glioma group $(p<0.0001)$, RPA classes $1-2(p<0.0001)$, first $\mathrm{RT}=60 \mathrm{~Gy}(p=0.0001)$, salvage surgery before SRS $(p=0.028)$, and other therapies before SRS $(p=0.017)$.

In multivariate analysis, only age $<40$ years and salvage surgery before SRS were associated with a 
Table 3 Patient and therapy characteristics

\begin{tabular}{|c|c|}
\hline \multicolumn{2}{|l|}{$\mathrm{Age}^{\mathrm{a}}$, years } \\
\hline Median & 51 \\
\hline Range & $18-79$ \\
\hline \multicolumn{2}{|l|}{ Gender, $n(\%)$} \\
\hline Female & $48(37.5)$ \\
\hline Male & $80(62.5)$ \\
\hline \multicolumn{2}{|l|}{$\mathrm{KPS}^{\mathrm{b}}, n(\%)$} \\
\hline$\leq 80$ & $118(92)$ \\
\hline$>80$ & $10(8)$ \\
\hline \multicolumn{2}{|c|}{ Initial WHO grade, $n(\%)$} \\
\hline IV grade & $88(69)$ \\
\hline III grade & $40(31)$ \\
\hline \multicolumn{2}{|l|}{ RPA classes, $n(\%)$} \\
\hline 1 & $18(14)$ \\
\hline 2 & $20(16)$ \\
\hline 4 & $31(24)$ \\
\hline 6 & $41(32)$ \\
\hline 7 & $18(14)$ \\
\hline \multicolumn{2}{|l|}{ Prior surgery ${ }^{\mathrm{c}}, n(\%)$} \\
\hline Biopsy & $11(10)$ \\
\hline Subtotal resection & $67(60)$ \\
\hline Gross total resection & $33(30)$ \\
\hline \multicolumn{2}{|c|}{ Prior radiotherapy ${ }^{\mathrm{d}}, n(\%)$} \\
\hline$<60$ Gy & $34(29)$ \\
\hline$\geq 60 \mathrm{~Gy}$ & $84(71)$ \\
\hline \multicolumn{2}{|c|}{ Prior chemotherapy ${ }^{\mathrm{d}}, n(\%)$} \\
\hline Yes & $84(72)$ \\
\hline No & $33(28)$ \\
\hline \multicolumn{2}{|c|}{ Salvage surgery (before SRS), $n(\%)$} \\
\hline Yes & $20(16)$ \\
\hline No & $108(84)$ \\
\hline \multicolumn{2}{|c|}{ Second-line chemotherapy (before SRS), $n(\%)$} \\
\hline Yes & $60(47)$ \\
\hline No & $68(53)$ \\
\hline \multicolumn{2}{|c|}{ Salvage surgery after SRS, $n(\%)$} \\
\hline Yes & $17(13)$ \\
\hline No & $111(87)$ \\
\hline \multicolumn{2}{|c|}{ Chemotherapy after SRS, $n(\%)$} \\
\hline Yes & $31(25)$ \\
\hline No & $91(75)$ \\
\hline
\end{tabular}

SRS radiosurgery

a Age at the time of reirradiation

${ }^{\mathrm{b}}$ KPS at the time of reirradiation

${ }^{c}$ Initial tumor resection (date of diagnosis)

${ }^{\mathrm{d}}$ Radiotherapy and chemotherapy treatment at diagnosis

significant improvement in survival. Figure 3 shows Kaplan-Meier curves of significant variables in multivariate analysis.
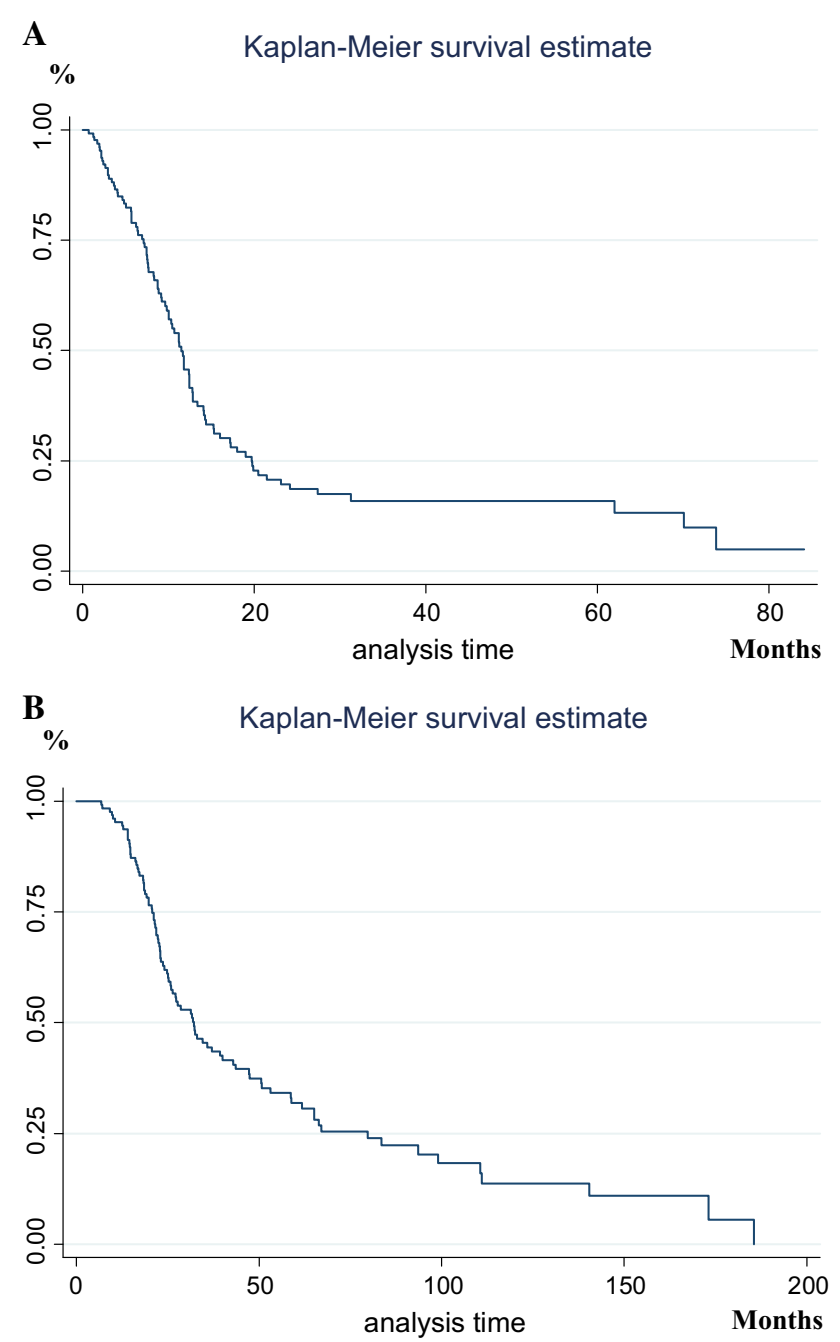

Fig. 2 Kaplan-Meier survival curves. a Overall survival for the entire patient population measured from the date of diagnosis to the date of death. b Overall survival for the entire patient population measured from the date of radiosurgery reirradiation to the date of death

\section{Survival from reirradiation}

Median survival following SRS was 11.5 months (95\% CI 9.8-12.7, SD \pm 0.9 ) (Fig. 2). The survival rate following SRS was 48, 20, and $17 \%$ at 1, 2, and 3 years, respectively. The median survival for patients younger than 40 years at diagnosis was 13 months (95 \% CI 10-19, $\mathrm{SD} \pm 0.6)$ and among patients $\geq 40$ years was 11 months (95\% CI 8-12, SD \pm 0.8 ). GBM and grade III glioma group had a median survival of 10 months (95\% CI 7.5-12, $\mathrm{SD} \pm 1)$ and 14 months (95\% CI 11-20, $\mathrm{SD} \pm 0.7)$, respectively.

The survival rate for patients $<40$ years old was 92 and $51 \%$ at 1 and 2 years, respectively for the grade III glioma subgroup, versus 38 and $19 \%$ for the GBM subgroup. 

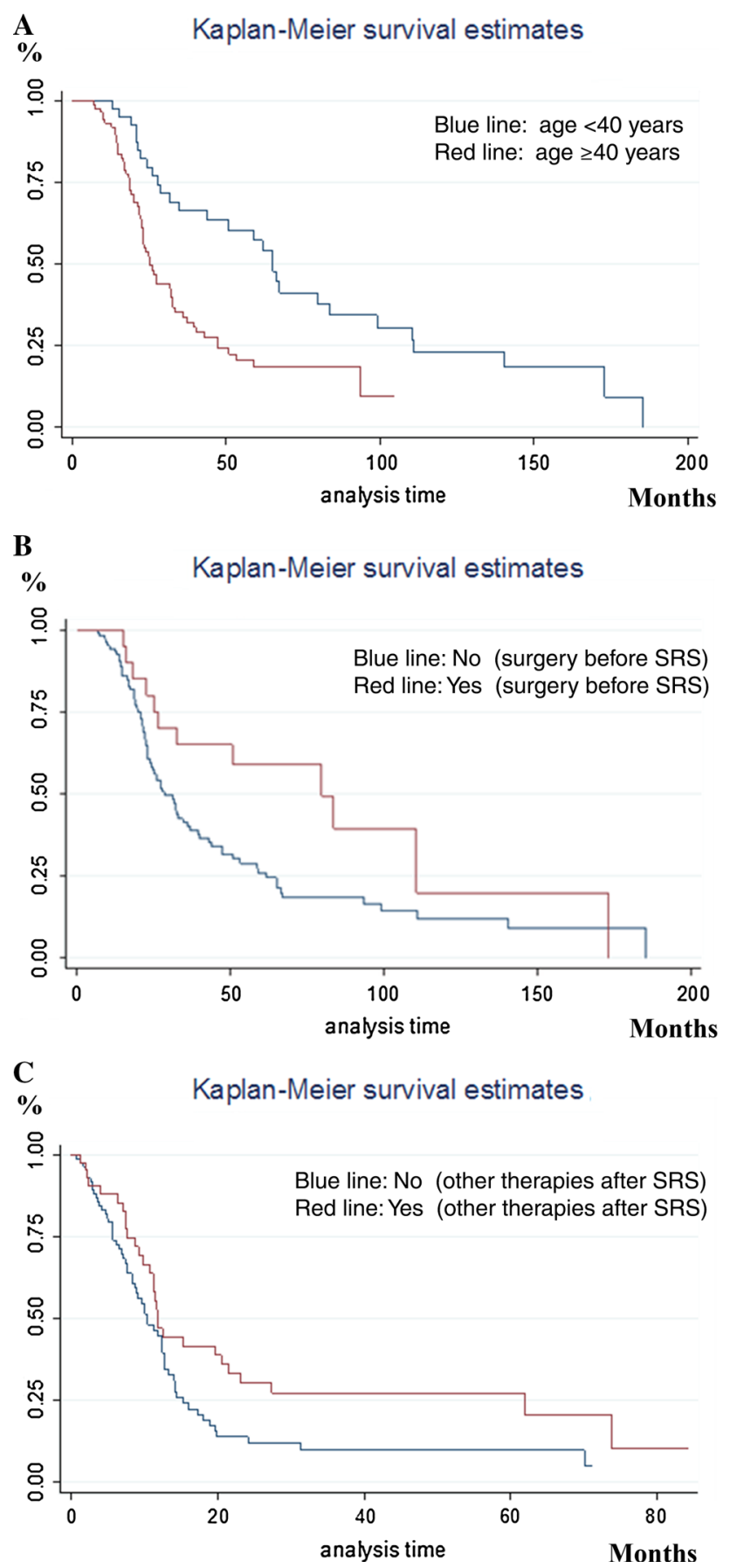

Fig. 3 Overall survival stratified by significant variables. a Overall survival from the date of diagnosis stratified by age at diagnosis (age $<40$ years vs $\geq 40$ years) showing significant dependence (univariate $p=0.0004$, multivariate $p=0.008$, HR 1.90). b Overall survival from the date of diagnosis stratified by salvage surgery before SRS reirradiation (yes vs no) showing significant dependence (univariate $p=0.0278$, multivariate $p=0.019$, HR 0.51). c Overall survival from the date of SRS reirradiation stratified by other therapy (chemotherapy and/or surgery) delivered after SRS reirradiation (yes vs no) showing significant dependence (univariate $p=0.0307$, multivariate $p=0.043$, HR 0.66)
Univariate analysis showed that age $<40$ years ( $p=0.046)$, grade III glioma group $(p=0.010)$, RPA classes $1-2 \quad(p=0.010)$, other therapies after SRS $(p=0.031), \mathrm{RT}=60 \mathrm{~Gy}(p=0.008)$ were associated with a significant improvement in survival. Lesion recurrence site (frontal lobe versus others) and volume had no impact on survival ( $p=0.4$ and $p=0.13$, respectively).

In multivariate analysis, only other post-SRS therapies (second-line chemotherapy and/or surgery) were found to significantly improve survival $(p=0.03)$. Figure 3 shows Kaplan-Meier curve of significant covariates at multivariate analysis. The details of statistical analysis are summarized in Table 2.

\section{Toxicity}

All patients completed SRS without interruption. Treatment compliance was good in all patients. Acute toxicity (grade $\leq 2$ for everyone), defined as headache, nausea, fatigue, and alopecia, was noted in 19 patients $(15 \%)$.

Radiation necrosis was observed in seven patients (6\%) with large volume lesions (diameter $>4 \mathrm{~cm}$ ) and was associated with neurological deterioration. The median time of radionecrosis presentation was 9 months (range 6-11 months). Three of these patients had surgical intervention with histological validation of radionecrosis.

\section{Discussion}

Therapeutic alternative options for r-HGG include surgery, chemotherapy, radiotherapy, and combined modalities. There is no clear evidence as to the superiority of one approach over another due to the absence of randomized studies [15]. Radiosurgery reirradation has been studied in literature and an evidence-based review of Tsao et al. [16] analyzed his role in HGG recurrence's setting. The authors concluded that there is insufficient evidence to support a survival benefit in the use of SRS for r-HGG compared with competing management strategies such as de-bulking surgery, chemotherapy, or best supportive care. Anyway the conclusions are limited by several biases, such as the wide variation in the employed technologies, lack of homogeneity in patients, and radiation doses. Generally reirradiation of r-HGG is limited by the higher risk of radiation-induced complications. Even when indicated, its application may be problematic due to the paucity of data concerning reirradiation: preferential size for re-treatment volumes, optimal time interval between first and second irradiation (most authors demand an interval of at least 6 months) [17-20], and the lack of reliable dose-volume 


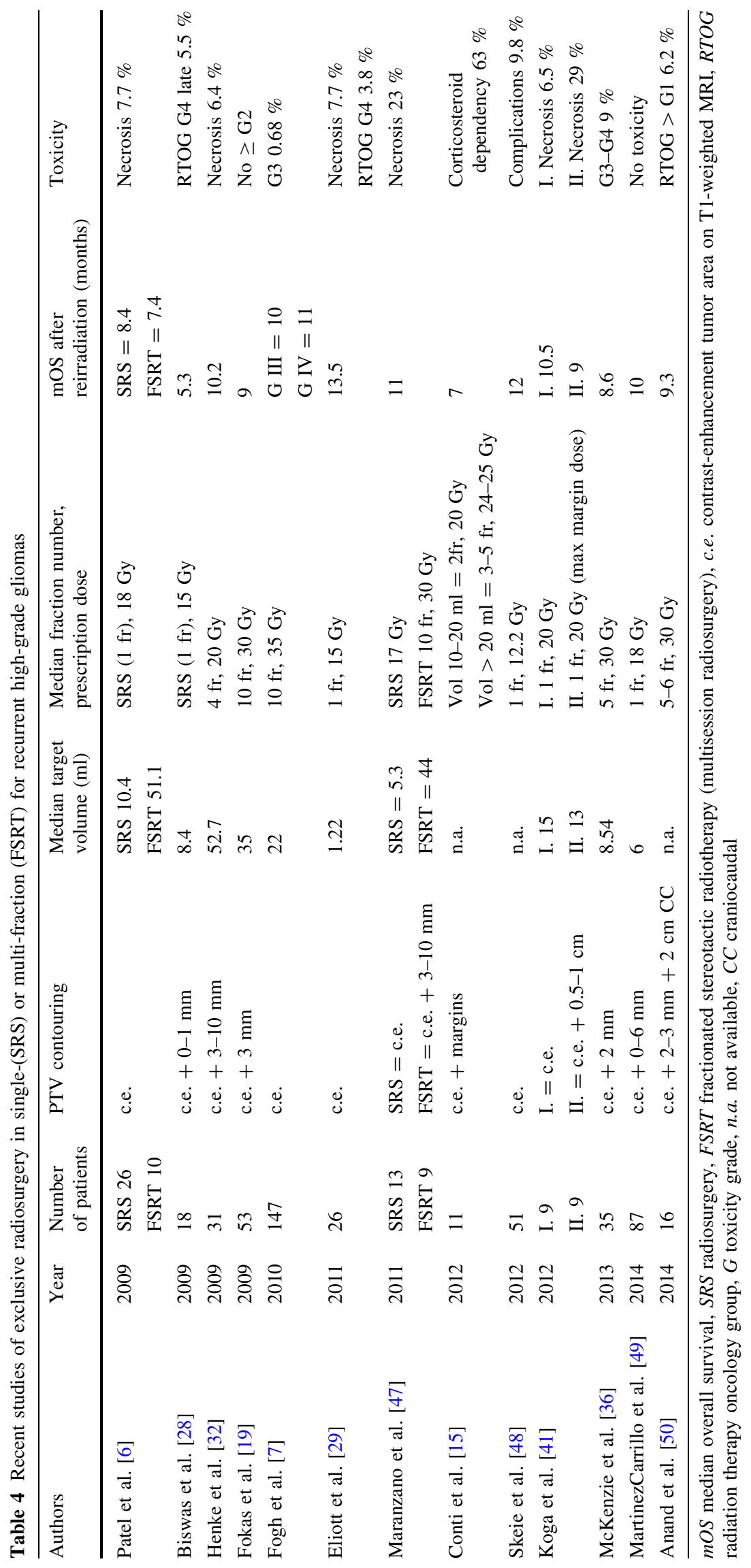


constraints. However, SRS is characterized by set-up accuracy and rapid radiation dose fall-off at the margins of treatment. SRS therefore reduces the radiation exposure of adjacent normal tissue. Several retrospective studies have demonstrated a median survival range of 7-17.9 months, although a selection bias in favor of patients who are amenable to SRS cannot be excluded [21-30]. Moreover, recent studies have described the effectiveness of salvage reirradiation for HGG recurrence or progression $[1,7,9$, $22,31-37]$, while also supporting the use of m-SRS in this patient population with minimal acute toxicity [33-35].

The present study analyzed a rather wide population of HGG high-dose reirradiation by means of single or multifraction radiosurgery. Two authors [33, 7], reporting on similar series, observed improved survival after HGG reirradiation, despite most patients received lower doses per fraction $(<4 \mathrm{~Gy})$. With regard to SRS as salvage therapy, our analysis confirmed these encouraging findings in terms of median survival and survival rate at 2 years. We found a median OS similar to that generally reported in the recent literature (Table 4): 32 months from diagnosis and 11.5 months after reirradiation (Fig. 2). With respect to analyzed variables, several authors found tumor grade, age at primary diagnosis, extent of resection, and volumes of reirradiation fields, to be predictors of OS [19, 38-41]. Our study confirmed statistically significant differences in survival only for patient's age. The tumor grade did not impact on survival $(p=0.883)$ most likely due to the fact that biomolecular characteristics could have a major impact on HGG patient's survival than basic histology. However, age $\leq 40$ years at diagnosis (initial surgery) affected survival from diagnosis. It is worth noting that to analyze the impact of age on survival we initially chose 50 years of age as a cut-off, as well as it was usually what literature referred. This value did not impact on survival. Finally, salvage surgery before SRS also improved survival from diagnosis $(p=0.019)$ as well as other therapies (secondline chemotherapy and/or surgery) delivered following SRS improved survival from SRS ( $p=0.043$ ) (Fig. 3).

These findings differ from our previously published results [42]: the cohort of analyzed patients could not be compared, as they also included outpatients with longer follow-up.

Several authors have found that the volume of reirradiated tumor correlates with survival [6, 21, 29, 43, 44]. Although we have considered three different cut-offs for tumor volumes (of an ideal spherical tumor volume): $7 \mathrm{~cm}^{3}$ (corresponding to $2.4 \mathrm{~cm}$ in diameter), $14 \mathrm{~cm}^{3}$ (corresponding to $3 \mathrm{~cm}$ in diameter), and $33.5 \mathrm{~cm}^{3}$ (corresponding to $4 \mathrm{~cm}$ in diameter), neither tumor volume nor the number of lesions and their location were found to be significantly related to survival. With respect to toxicity, the radionecrosis data reported in the literature indicate an average of $5-14 \%[16,45,46]$. Otherwise, Fokas et al. [19] did not report high toxicity despite the fact that median tumor volume $\left(35 \mathrm{~cm}^{3}\right)$ was double than the volumes reported in previous studies, at a higher dose than the average reported in the literature. We also observed a low overall toxicity: only 7 (6\%) grade 3-4 neurological adverse events could be related to large s-SRS irradiated volumes. This may support the hypothesis that m-SRS could be a suitable option in difficult reirradiations, such as lesions larger than $4 \mathrm{~cm}$ or in the eloquent cortex.

Thus, the high dose delivered by reirradiation SRS (single- and multi-fraction) involves a low toxicity profile and an encouraging outcome.

\section{Conclusions}

In our study, the large tumor volumes and number of lesions represented no obstacle for radiosurgical treatment as long as the dose was fractionated in order to prevent toxicity.

The question should no longer be whether or not reirradiation is a valid treatment option, but when and which salvage treatments should be administered in prospective trials.

Acknowledgments The authors thank Dr. A. Harrington for reviewing the English of the manuscript and Milda Cerniauskaite for her collaboration.

Conflict of interest The authors state that there are no conflicts of interest.

\section{References}

1. Combs SE, Gutwein S, Thilmann C et al (2005) Stereotactically guided fractionated re-irradiation in recurrent glioblastoma multiforme. J Neurooncol 74(2):167-171

2. Darakchiev BJ, Albright RE, Breneman JC et al (2008) Safety and efficacy of permanent iodine-125 seed implants and carmustine wafers in patients with recurrent glioblastoma multiforme. J Neurosurg 108(2):236-242

3. Mamelak AN, Rosenfeld S, Bucholz R et al (2006) Phase I single-dose study of intracavitary-administered iodine-131-TM-601 in adults with recurrent high-grade glioma. J Clin Oncol 24(22):3644-3650

4. Stupp R, Wong ET, Kanner AA et al (2012) NovoTTF100Aversus physician's choice chemotherapy in recurrent glioblastoma: arandomised phase III trial of a novel treatment modality. Eur J Cancer 48:2192-2202

5. Butowski NA, Sneed PK, Chang SM (2006) Diagnosis and treatment of recurrent high-grade astrocytoma. J Clin Oncol 24(8):1273-1280

6. Patel M, Siddiqui F, Jin JY et al (2009) Salvage reirradiation for recurrent glioblastoma with radiosurgery: radiographic response and improved survival. J Neurooncol 92(2):185-191

7. Fogh SE, Andrews DW, Glass J et al (2010) Hypofractionated stereotactic radiation therapy: an effective therapy for recurrent high-grade gliomas. J Clin Oncol 28(18):3048-3053 
8. Kirkpatrick JP, Sampson JH (2014) Recurrent malignant gliomas. Semin Radiat Oncol 24(4):289-298

9. Mayer R, Sminia P (2008) Reirradiation tolerance of the human brain. Int J Radiat Oncol Biol Phys 70(5):1350-1360

10. Jones B, Dale RG, Finst P, Khaksar SJ (2000) Biological equivalent dose assessment of the consequences of hypofractionated radiotherapy. Int $\mathrm{J}$ Radiat Oncol Biol Phys 47(5):1379-1384

11. Qi XS, Schultz CJ, Li XA (2006) An estimation of radiobiologic parameters from clinical outcomes for radiation treatment planning of brain tumor. Int J Radiat Oncol Biol Phys 64(5):1570-1580

12. Cooperative Group Common Toxicity Criteria, Neurologic. http://www.rtog.org/ResearchAssociates/AdverseEventReporting/ CooperativeGroupCommonToxicityCriteria.aspx. Accessed 14 Oct 2014

13. Cancer Therapy Evaluation Program (CTEP), Common Terminology Criteria for Adverse Events (CTCAE) v3.0. http://ctep. cancer.gov/protocolDevelopment/electronic_applications/docs/ ctcaev3.pdf. Accessed 14 Oct 2014

14. Carson KA, Grossman SA, Fisher JD, Shaw EG (2007) Prognostic factors for survival in adult patients with recurrent glioma enrolled onto the new approaches to brain tumor therapy CNS consortium phase I and II clinical trials. J Clin Oncol 25(18):2601-2606

15. Conti A, Pontoriero A, Arpa D et al (2012) Efficacy and toxicity of CyberKnife re-irradiation and "dose dense" temozolomide for recurrent gliomas. Acta Neurochir (Wien) 154(2):203-209

16. Tsao MN, Mehta MP, Whelan TJ et al (2005) The American Society for Therapeutic Radiology and Oncology (ASTRO) evidence-based review of the role of radiosurgery for malignant glioma. Int J Radiat Oncol Biol Phys 63(1):47-55

17. Ernst-Stecken A, Ganslandt O, Lambrecht U et al (2007) Survival and quality of life after hypofractionated stereotactic radiotherapy for recurrent malignant glioma. J Neurooncol 81(3):287-294

18. Combs SE, Debus J, Schulz-Ertner D (2007) Radiotherapeutic alternatives for previously irradiated recurrent gliomas. BMC Cancer 7:167

19. Fokas E, Wacker U, Gross MW et al (2009) Hypofractionated stereotactic reirradiation of recurrent glioblastomas : a beneficial treatment option after high-dose radiotherapy? Strahlenther Onkol 185(4):235-240

20. Niyazi M, Söhn M, Schwarz SB et al (2012) Radiation treatment parameters for re-irradiation of malignant glioma. Strahlenther Onkol 188(4):328-333

21. Shrieve DC, Alexander E 3rd, Wen PY et al (1995) Comparison of stereotactic radiosurgery and brachytherapy in the treatment of recurrent glioblastoma multiforme. Neurosurgery 36(2):275-282 (discussion 282-284)

22. Cho KH, Hall WA, Gerbi BJ et al (1999) Single dose versus fractionated stereotactic radiotherapy for recurrent high-grade gliomas. Int J Radiat Oncol Biol Phys 45(5):1133-1141

23. Kondziolka D, Flickinger JC, Bissonette DJ et al (1997) Survival benefit of stereotactic radiosurgery for patients with malignant glial neoplasms. Neurosurgery 41(4):776-783 (discussion 783-785)

24. Park KJ, Kano H, Iyer A et al (2012) Salvage gamma knife stereotactic radiosurgery followed by bevacizumab for recurrent glioblastoma multiforme: a case-control study. J Neurooncol 107(2):323-333

25. Larson DA, Prados M, Lamborn KR et al (2002) Phase II study of high central dose Gamma Knife radiosurgery and marimastat in patients with recurrent malignant glioma. Int J Radiat Oncol Biol Phys 54(5):1397-1404

26. Combs SE, Widmer V, Thilmann C et al (2005) Stereotactic radiosurgery (SRS): treatment option for recurrent glioblastoma multiforme (GBM). Cancer 104(10):2168-2173
27. Mahajan A, McCutcheon IE, Suki D et al (2005) Case-control study of stereotactic radiosurgery for recurrent glioblastoma multiforme. J Neurosurg 103(2):210-217

28. Biswas T, Okunieff P, Schell MC et al (2009) Stereotactic radiosurgery for glioblastoma: retrospective analysis. Radiat Oncol 4:11

29. Elliott RE, Parker EC, Rush SC et al (2011) Efficacy of gamma knife radiosurgery for small-volume recurrent malignant gliomas after initial radical resection. World Neurosurg 76(1-2):128-140

30. Park KJ, Kano H, Iyer A et al (2012) Salvage gamma knife stereotactic radiosurgery followed by bevacizumab for recurrent glioblastoma multiforme: a case-control study. J Neurooncol 107(2):323-333

31. Hudes RS, Corn BW, Werner-Wasik M et al (1999) A phase I dose escalation study of hypofractionated stereotactic radiotherapy as salvage therapy for persistent or recurrent malignant glioma. Int J Radiat Oncol Biol Phys 43(2):293-298

32. Henke G, Paulsen F, Steinbach JP et al (2009) Hypofractionated reirradiation for recurrent malignant glioma. Strahlenther Onkol 185(2):113-119

33. Combs SE, Thilmann C, Edler L, Debus J, Schulz-Ertner D (2005) Efficacy of fractionated stereotactic reirradiation in recurrent gliomas: long-term results in 172 patients treated in a single institution. J Clin Oncol 3(34):8863-8869

34. Vordermark D, Kölbl O, Ruprecht K et al (2005) Hypofractionated stereotactic re-irradiation: treatment option in recurrent malignant glioma. BMC Cancer 5:55

35. Combs SE, Gutwein S, Thilmann C et al (2005) Reirradiation of recurrent WHO grade III astrocytomas using fractionated stereotactic radiotherapy (FSRT). Strahlenther Onkol 181(12):768-773

36. McKenzie JT, Guarnaschelli JN, Vagal AS et al (2013) Hypofractionated stereotactic radiotherapy for unifocal and multifocal recurrence of malignant gliomas. J Neurooncol 113(3):403-409

37. Villavicencio AT, Burneikiene S, Romanelli P et al (2009) Survival following stereotactic radiosurgery for newly diagnosed and recurrent glioblastoma multiforme: a multicenter experience. Neurosurg Rev 32(4):417-424

38. Mehta MP, Masciopinto J, Rozental J et al (1994) Stereotactic radiosurgery for glioblastoma multiforme: report of a prospective study evaluating prognostic factors and analyzing long-term survival advantage. Int J Radiat Oncol Biol Phys 30(3):541-549

39. Scholtyssek F, Zwiener I, Schlamann A et al (2013) Reirradiation in progressive high-grade gliomas: outcome, role of concurrent chemotherapy, prognostic factors and validation of a new prognostic score with an independent patient cohort. Radiat Oncol $8(1): 161$

40. Weller M, Cloughesy T, Perry JR, Wick W (2013) Standards of care for treatment of recurrent glioblastoma-are we there yet? Neuro Oncol 15(1):4-27

41. Koga T, Maruyama K, Tanaka M et al (2012) Extended field stereotactic radiosurgery for recurrent glioblastoma. Cancer 118(17):4193-4200

42. Nava F, Tramacere I, Fittipaldo A et al (2014) Survival effect of first- and second-line treatments for patients with primary glioblastoma: a cohort study from a prospective registry, 1997-2010. Neuro Oncol 16(5):719-727

43. Pouratian N, Crowley RW, Sherman JH, Jagannathan J, Sheehan JP (2009) Gamma Knife radiosurgery after radiation therapy as an adjunctive treatment for glioblastoma. J Neurooncol 94(3):409-418

44. Kong DS, Lee JI, Park K et al (2008) Efficacy of stereotactic radiosurgery as a salvage treatment for recurrent malignant gliomas. Cancer 112(9):2046-2051

45. Hall WA, Djalilian HR, Sperduto PW et al (1995) Stereotactic radiosurgery for recurrent malignant gliomas. J Clin Oncol 13(7):1642-1648 
46. Nieder C, Grosu AL, Molls M (2000) A comparison of treatment results for recurrent malignant gliomas. Cancer Treat Rev 26(6):397-409

47. Maranzano E, Anselmo P, Casale M et al (2011) Treatment of recurrent glioblastoma with stereotactic radiotherapy: long-term results of a mono-institutional trial. Tumori 97(1):56-61

48. Skeie BS, Enger PO, Brøgger J et al (2012) Gamma knife surgery versus reoperation for recurrent glioblastoma multiforme. World Neurosurg 78(6):658-669
49. Martínez-Carrillo M, Tovar-Martín I, Zurita-Herrera $M$ et al (2014) Salvage radiosurgery for selected patients with recurrent malignant gliomas. Biomed Res Int 2014:657953. doi:10.1155/ 2014/657953 (In press)

50. Anand AK, Kumar P, Patir R et al (2014) Fractionated stereotactic radiosurgery with volumetric modulated arc therapy (Rapid Arc) for reradiation in recurrent high grade gliomas. J Cancer Res Ther 10(1):97-102 